
Revue
de l'histoire
Revue de l'histoire des religions
des religions
$3 \mid 2009$
La culture gallicane. Références et modèles (droit, ecclésiologie, histoire)

\title{
Dionysisme et gallicanisme : la figure de l'évêque selon Bossuet
}

Gallicanism and Dionysius'influence: the bishop's figure in Bossuet's works

\section{Anne Régent-Susini}

\section{CpenEdition}

\section{Journals}

Édition électronique

URL : http://journals.openedition.org/rhr/7272

DOI : $10.4000 /$ rhr.7272

ISSN : 2105-2573

Éditeur

Armand Colin

Édition imprimée

Date de publication : 1 juillet 2009

Pagination : 413-428

ISBN : 978-2200-92591-8

ISSN : 0035-1423

Référence électronique

Anne Régent-Susini, « Dionysisme et gallicanisme : la figure de l'évêque selon Bossuet », Revue de I'histoire des religions [En ligne], 3 | 2009, mis en ligne le 01 juillet 2012, consulté le 19 avril 2019. URL http://journals.openedition.org/rhr/7272 ; DOI : 10.4000/rhr.7272 


\section{Dionysisme et gallicanisme : la figure de l'évêque selon Bossuet}

La valorisation de la figure épiscopale revêt, on le sait, une importance décisive dans le gallicanisme en général, et dans le gallicanisme de Bossuet en particulier. Fortement marquée par le concept dionysien du hiérarque, la pensée de l'épiscopat développée par Bossuet fait cependant subir à cet héritage un infléchissement notable : minoration du rôle joué par les corps intermédiaires (en l'occurrence les prêtres, et surtout les diacres), interprétation de la relation hiérarchique comme une relation d'autorité plus que de transmission d'un savoir, importance accordée au statut paradoxal de l'évêque, oscillant entre force et faiblesse, majesté et humilité.

\section{Gallicanism and Dionysius'influence : the bishop's figure in Bossuet's works}

It is well known that the promotion of the bishop figure is of primary importance to gallicanism in general, and to Bossuet's gallicanism in particular. Strongly influenced by Dionysius 'concept of hierarch, Bossuet's conception of episcopate shows some remarkable differences with this heritage: Indeed, Bossuet decreases the importance of the intermediate bodies (such as the priests, and most of all the deacons); he views the hierarchy as an authoritative relationship, and not as a transmission of knowledge; and he tends to emphasize the paradoxical nature of the bishop's situation, always oscillating between strengh and weakness. 
La valorisation de la figure épiscopale constitue, on le sait, un aspect important du gallicanisme en général, et du gallicanisme de Bossuet en particulier. En tant que telle, elle a été étudiée en son temps par Aimé-Georges Martimort dans son Gallicanisme de Bossuet - thèse certes partiale et contestable par endroits ${ }^{1}$, mais qui demeure, à bien des égards, précieuse. Il ne s'agira donc pas ici de reprendre les questions relatives à la pensée bossuétiste de l'épiscopat déjà traitées par Martimort, en particulier la manière complexe dont, chez Bossuet, l'autorité épiscopale s'articule avec l'autorité romaine d'une part, avec l'autorité monastique d'autre part. Il ne s'agira pas davantage de s'attacher à la pratique épiscopale de Bossuet (évêque de Condom et surtout de Meaux), scrutée tout récemment par Michèle Bardon dans une synthèse intitulée Bossuet à Meaux. La vie de Bossuet dans son diocèse $e^{2}$. On se focalisera bien plutôt sur la représentation de l'évêque telle qu'elle se dégage de la lecture de l'œuvre de Bossuet et sur l'appropriation de l'héritage dionysien dont elle témoigne.

S'intéresser à la figure de l'évêque dans sa singularité constitue en soi une démarche significative, dans la mesure où elle accorde au ministère épiscopal une certaine spécificité. Or, cette spécificité fait justement, au XVII ${ }^{\mathrm{e}}$ siècle, l'objet d'un débat : l'épiscopat est-il un sacrement distinct de la prêtrise? Ou n'est-il qu'une sorte d'extension, d'accomplissement du sacerdoce ${ }^{3}$ ? Cette ambivalence est très

1. Voir Jean-Louis Quantin, "Bossuet et l'érudition de son temps », Gérard Ferreyrolles (dir.), Bossuet. Le Verbe et l'Histoire (1704-2004). Actes du colloque international de Paris et de Meaux pour le troisième centenaire de la mort de Bossuet, Paris, Honoré Champion, 2006, p. 65-103, et plus largement, la conférence tenue par le même chercheur sur le même thème en 2003-2004 à l'École pratique des Hautes Études.

2. Michèle Bardon, Bossuet à Meaux. La vie de Bossuet dans son diocèse, Étrépilly, Les Presses du Village, 2004. M. Bardon y exploite notamment, outre les œuvres et la correspondance de Bossuet, des sources plus rarement sollicitées telles que les procès-verbaux des visites pastorales effectuées par Bossuet et divers apports documentaires régionaux (revues et archives de la Seine-et-Marne). Plus largement, l'épiscopat français dans les décennies qui ont immédiatement précédé le règne de Louis XIV a été remarquablement étudié par Joseph Bergin dans son étude intitulée The Making of the French Episcopate, 1589-1661, New Haven et Londres, Yale University Press, 1996.

3. Voir Michel Péronnet, Les Évêques de l'ancienne France, Paris, Honoré Champion, 1977, t. I, p. 387. 
sensible chez Bossuet, qui considère l'évêque, d'une part, comme le prêtre par excellence, sans qu'il y ait entre lui et le simple prêtre de solution de continuité - comme en témoigne le Panégyrique de saint Charles Borromée tel qu'il fut pris à l'audition, vraisemblablement par un tachygraphe :

« je remarque dans tous les prêtres de la nouvelle alliance cette obligation, et particulièrement dans les évêques, qui portent éminemment cette dignité du sacerdoce, qui enferme en soi trois admirables qualités. Car les prêtres sont les intercesseurs pour le peuple; ils sont les princes du peuple; ils sont les pères du peuple ${ }^{4} »$.

"Particulièrement dans les évêques " : 1'évêque est un prêtre prêtre éminent certes, mais prêtre avant tout, partageant les mêmes obligations que les autres prêtres. Toutefois, Bossuet réaffirme à maintes reprises la spécificité de la mission épiscopale. Il se montre alors profondément marqué par l'ecclésiologie gallicane, dans laquelle l'évêque tient une place centrale, fortifiée et clarifiée par la réforme tridentine, qui attribue à l'évêque un rôle clé dans l'établissement de la discipline nouvelle imposée aux ecclésiastiques - et de fait, la figure épiscopale apparaît fréquemment liée, chez Bossuet, au maintien de la discipline. Rien de très surprenant à cela : on sait l'interprétation particulière que fit l'épiscopat français, soutenu par Louis XIV, de la revalorisation des évêques encouragée par le concile de Trente, en l'articulant aux traditions gallicanes touchant à la dignité et à l'importance du ministère épiscopal. Dans le Sermon sur l'unité de l'Église, Bossuet se réjouit de ce nouveau climat, et affirme que l'autorité nouvelle des évêques est soutenue par le pouvoir temporel comme par les trouvailles nouvelles de la théologie positive :

«La main de Louis était réservée pour achever de guérir les plaies de l'Église. Déjà celles de l'épiscopat ne nous paraissent plus irrémédiables. Outre cent arrêts favorables, sous les auspices d'un prince qui ne veut que voir la raison pour s'y soumettre, on ouvre les yeux : on ne lit plus les canons et les décrets des saints Pères par pièces et par lambeaux pour nous y tendre des pièges; on prend la suite des antiquités ecclésiastiques; et si on entre dans cet esprit, que verra-t-on à toutes les pages, que des monuments éternels de notre autorité sacrée $?^{5}{ }^{\prime}$

4. Bossuet, Panégyrique de saint Charles, pris à l'audition, in O.O. (pour Euvres oratoires, éditées par Ch. Urbain et É. Levesque, 7 t., Paris, Desclée de Brouwer, 1911-1926), t. II, p. 577.

5. Bossuet, Sermon sur l'Unité de l'Église (1681), in O.O., t. VI, p. 144. 
La question demeure donc en suspens : le ministère épiscopal, simple prolongement du ministère sacerdotal ou ministère spécifique? Bossuet ne paraît pas trancher - même si la place singulière occupée dans son œuvre par la figure épiscopale semble peser en faveur de la seconde hypothèse.

Quoi qu'il en soit, une certitude: l'épiscopat se situe pour Bossuet au cœur de la fonction d'intercession de l'Église - dans une perspective marquée à bien des égards par le dionysisme, si répandu au XVII ${ }^{\mathrm{e}}$ siècle, par l'intermédiaire de saint Thomas notamment. On sait en effet que, très forte au Moyen Âge ${ }^{6}$ l'influence dionysienne survit sans trop de mal aux progrès philologiques et critiques de la Renaissance et du premier XVII ${ }^{\mathrm{e}}$ siècle, en dépit des problèmes de plus en plus grands que pose la figure du pseudoDenys, superposition mythique de trois figures « historiques » : le magistrat de l'Aréopage converti par saint Paul dans les Actes des Apôtres, l'auteur du corpus dionysien et le premier évêque de Paris, martyrisé au $\mathrm{III}^{\mathrm{e}}$ siècle, considéré comme l'« apôtre de la France »?. Même si pour Bossuet, écrivain de la seconde moitié du siècle, cet héritage s'avère plus problématique qu'il ne l'était juste après le Concile de Trente ${ }^{8}$, le constat de Jacques Le Brun selon lequel « le

6. L'influence du Pseudo-Denys sur la théologie médiévale, et notamment sur les docteurs de Sorbonne, a donné lieu à plusieurs études récentes, parmi lesquelles on peut citer : Tzotcho Boiadjiev, Georgi Kapriev et Andreas Speer (dir.), Die Dionysius-Rezeption im Mittelalter, Turnhout, Brepols, 2000; et L. Michael Harrington, A Thirteenth-Century Textbook of Mystical Theology at the University of Paris, Leuven, Peeters, 2004. Voir aussi Hyacinthe-François Dondaine, Le Corpus dionysien de l'université de Paris au XIII siècle, Rome, Edizioni di Storia e Letteratura, 1953; et Josef Pieper, Scholasticism. Personalities and Problems of Medieval Philosophy, Londres, Faber \& Faber 1961, p. 46 et suiv. Voir aussi, dans une perspective plus générale, Ysabel De Andia, Denys l'Aréopagite et sa postérité en Orient et en Occident, Paris, Institut d'Études Augustiniennes, 1997; et Jean-Marie Le Gall, Le Mythe de saint Denis entre Renaissance et Révolution, Seyssel, Champ Vallon, 2007.

7. G. Ferreyrolles cite pour exemple de cette dénomination, fréquente dans les textes du début du XVII ${ }^{\mathrm{e}}$ siècle, La Vie apostolique de saint Denys Aréopagite et apôtre de la France, par Étienne Binet, 1624. De même, plus proche de Bossuet, saint François de Sales, lui-même imprégné de pensée dionysienne, parle du « grand apôtre de France » (« Pascal et Denys l'Aréopagite », Rudolf Behrens, Andreas Gipper et Viviane Mellinghoff-Bourgerie (dir.), Croisements d'anthropologies. Pascals Pensées im Geflecht der Anthropologien, Heidelberg, Universitätverlag, 2005, p. 183-202).

8. En dehors des problèmes proprement historiques et critiques posés par la figure syncrétique et partiellement mythique du Pseudo-Denys, le déclin de la figure dionysienne s'explique également par des raisons proprement littéraires, le 
dionysisme est au XVII siècle le caractère le plus constant de la théologie de l'Église $"^{9} \mathrm{~s}$ 'applique tout particulièrement à l'évêque de Meaux, qui ne peut se montrer totalement insensible à la récupération nationale dont fait l'objet depuis longtemps la troisième facette de la figure dionysienne (Denys évêque martyr de Paris) - ce dont témoigne tout particulièrement le Sermon dit sur l'unité de l'Église, voix de l'Église gallicane, dont la coloration dionysienne est très marquée.

Cela n'équivaut pas pour autant à dire que le pseudo-Denys constitue chez Bossuet une autorité essentiellement nationale et gallicane. Il est aussi - voire surtout - une autorité caractéristique de la Réforme catholique et c'est bien sûr avant tout dans cette perspective que doit se comprendre l'influence qu'il exerce sur l'ecclésiologie de Bossuet, et en particulier sur sa conception de l'épiscopat. Face aux attaques protestantes contre les dévoiements humains de l'Église, face, aussi, à la promotion nouvelle d'une relation directe de l'individu avec Dieu, le corpus dionysien permettait en effet de mettre l'accent sur l'analogie entre hiérarchie ecclésiastique et hiérarchie céleste, et par là de sacraliser pleinement l'Église, au lieu de l'envisager comme institution proprement humaine ${ }^{10}$. En outre, le principe de la médiation qui fondait la conception dionysienne du monde offrait un soutien opportun aux défenseurs des cultes des saints et de la Vierge, et à la promotion catholique d'une transmission de l'Écriture par la double médiation de la tradition et des institutions ecclésiastiques. Bossuet hérite donc d'une tradition alors relativement récente utilisant le corpus dionysien dans la controverse anti-protestante - et c'est tout d'abord dans cette

style de la traduction donnée par Goulu du corpus aéropagitique n'étant guère conforme aux canons de la langue théologique classique; voir sur ce point G. Ferreyrolles, «Pascal et Denys l'Aréopagite », ibid.

9. Jacques Le Brun, La Spiritualité de Bossuet, Paris, Klincksieck, 1970, p. 159, n. 443. De même, Jean Orcibal constate que les catholiques du XVII ${ }^{\mathrm{e}}$ siècle ne traitaient jamais la question de l'Église « sans se référer, fût-ce inconsciemment, au plus vénéré des Pères apostoliques, le pseudo-Denys l'Aréopagite " ("L'idée d'Église chez les catholiques du XVII ${ }^{\mathrm{e}}$ siècle », in Études d'histoire et de littérature religieuse, $X V I^{e}-X V I I I^{e}$ siècle, éd. Jacques Le Brun et Jean Lesaulnier, Paris, Klincksieck, 1997, p. 339). Même un auteur aussi tardif que Fénelon, souvent associé à une forme d'individualisme religieux, professe encore une ecclésiologie hiérarchique et collective se situant dans la stricte lignée dionysienne, autour des thèmes fondateurs de l'unité et de la médiation.

10. Sur la pensée de la hiérarchie que Bossuet hérite du pseudo-Denys, voir J. Le Brun, La Spiritualité de Bossuet, op. cit., p. 100-106. 
optique qu'il lit la Hiérarchie ecclésiastique au début de sa carrière, dans les années $1660^{11}$.

\section{UN RELAIS ESSENTIEL : BÉRULLE}

Cet héritage passe par un intermédiaire majeur: il s'agit de Bérulle, relais capital du dionysisme au cours de la première moitié du siècle. On sait en effet que c'est sur les auteurs composant, selon la très contestée typologie de Bremond, l' '«École française de spiritualité $\gg{ }^{12}$, que l'influence dionysienne est alors la plus évidente : Chardon (traducteur de La Théologie mystique), Benoît de Canfield, Saint-Jure, Olier, Surin, et bien sûr Bérulle ${ }^{13}$. Représentatif de toute la génération postérieure au concile de Trente et exaltant une conception du sacerdoce fortement influencée par le Corpus Aeropagiticum $^{14}$, Bérulle constitue un intermédiaire capital dans la réception que fait Bossuet du dionysisme, et il transmet en particulier au futur évêque de Meaux la figure à la fois très haute et très exigeante $d u$ « hiérarque dionysien $»^{15}$, qui répand la lumière

\section{J. Le Brun, La Spiritualité de Bossuet, p. 161.}

12. On sait ce que cette appellation, et le groupement d'auteurs auquel elle renvoie, présente de problématique et de contestable. Il semble cependant que l'influence dionysienne s'exerce, à des degrés divers et selon des modalités différentes, sur tous ces auteurs.

13. Si c'est sur Bérulle que l'empreinte dionysienne paraît à bien des égards la mieux connue, J. Le Brun souligne que des études restent à mener sur la spécificité de cet héritage: "Les travaux récents insistent beaucoup sur ce dionysisme de Bérulle et renouvellent l'idée que nous nous faisions de sa spiritualité : l'enquête devra porter maintenant sur son originalité; en un temps où le dionysisme est si répandu, pourquoi conduit-il Bérulle si loin de ses contemporains?» (J. Le Brun, art. «France. XVII ${ }^{\mathrm{e}}$ siècle; le Grand siècle de la spiritualité française et ses lendemains », Dictionnaire de spiritualité ascétique et mystique, t. 5, col. 933).

14. C'est ainsi qu'un François de La Rochefoucauld, dans son traité De l'estat ecclesiastique (1597), introduit son développement sur la vocation sacerdotale par une évocation majestueuse de la hiérarchie des créatures. D'autres auteurs, plus proches de Bérulle, insistent davantage sur la dignité du sacerdoce et l'exigence de piété profonde qu'il implique.

15. Sur la place du pseudo-Denys dans la spiritualité sacerdotale du XVII ${ }^{\mathrm{e}}$ siècle, en particulier chez Bérulle, voir Paul Cochois, «Bérulle, hiérarque dionysien », Revue d'ascétique et de mystique, 1961, p. 314-353, et Michel Dupuy, Bérulle et le sacerdoce. Étude historique et doctrinale. Textes inédits, Paris, Lethielleux («Bibliothèque d'histoire et d'archéologique chrétienne »), 1969. Il est à noter que Bérulle n'a pas laissé de traité concernant le ministère du prêtre, mais que sa conception du sacerdoce trouve une exposition particulièrement remarquable dans 
divine sur tous les ordres inférieurs et diffuse ainsi l'unité dans la multiplicité, avant de retourner aussitôt au principe divin dont il procède et qui le sanctifie (c'est d'ailleurs ainsi que Bérulle conçoit sa propre fonction à la tête de l'Oratoire). Pour Bérulle comme pour le pseudo-Denys, et pour Bossuet comme pour Bérulle, le hiérarque, « vivant dans le commerce habituel des réalités saintes, il doit être lui-même saint $\gg^{16}$ : instrument de purification, d'illumination et de perfection ${ }^{17}$ - car telles sont les trois fonctions que le pseudo-Denys lui attribue, il doit faire «monter l'ensemble de ceux qui lui sont confiés dans la hiérarchie mystique des âmes $\|^{18}$.

Fortement marqué par la spiritualité bérullienne en général, Bossuet se montre donc tout spécialement imprégné de la revalorisation du sacerdoce effectuée par le fondateur de l'Oratoire, comme en témoigne avec éclat l'Oraison funèbre du Père Bourgoing, supérieur de l'Oratoire. Au-delà même de la controverse anti-protestante ${ }^{19}$, le pseudo-Denys constitue un socle important de son ecclésiologie, telle qu'elle s'exprime notamment dans la $I V^{e}$ lettre à une demoiselle de $\mathrm{Metz}^{20}$, et les thèmes de l'unité21 et de l'harmonie ordonnée du monde trouvent chez lui, comme chez le pseudo-Denys, un retentissement à la fois spirituel, intellectuel et esthétique ${ }^{22}$. On retrouve en

un livre de son disciple Paul Metezeau, De sancto sacerdotio, eiusque dignitate et functionibus sacris, Paris, 1631. Voir l'Histoire du christianisme des origines à nos jours, t. VIII, « Le temps des confessions (1530-1620/30), dir. Marc Venard, Paris, Desclée, 1992, $3^{\mathrm{e}}$ partie, « La vie des chrétiens », chap. I, «Les clergés », V. Barrie-Currien et M. Venard, p. 875.

16. René Roques, L'Univers dionysien. Structure hiérarchique du monde selon le Pseudo-Denys, Paris, Cerf, 1983, p. 182.

17. Voir Yves Durand, «Mystique et politique au XVII siècle : l'influence du Pseudo-Denys », XVII siècle, no 173, oct-déc. 1991, p. 333.

18. Yves Krumenacker, L'École française de spiritualité. Des mystiques, des fondateurs, des courants et leurs interprètes, Paris, Cerf, 1998, p. 161.

19. Comme le soulignent les notes qu'il prend sur son édition : "Consecrari et consecrare, illustrari et illustrare, perfici et perficere » (voir J. Le Brun, La Spiritualité de Bossuet, p. 161).

20. Voir J. Le Brun, La Spiritualité de Bossuet, p. 162.

21. Ce thème fascinait déjà Bérulle. Voir Y. Krumenacker, op. cit., p. 176 sq.

22. Même si, comme le souligne J. Le Brun, Bossuet illustre le changement radical de statut que connaît dès les années 1650 la conception dionysienne de l'univers, toute en communication, en harmonie et en médiation : « Cette théologie, qui exerça une telle influence au XVI ${ }^{\mathrm{e}}$ siècle et au début du XVII ${ }^{\mathrm{e}}$ siècle, ne correspond plus du tout à l'état des esprits au temps de Bossuet : de synthèse unificatrice, elle devient discipline particulière, un des éléments du champ du savoir; ses schémas n'organisent plus les sciences du monde, de l'astronomie à la médecine; les choses et les êtres ne se définissent plus par leur «situation » par rapport à l'ensemble 
effet chez Bossuet les quatre motifs principaux qui composent selon Yves Congar ${ }^{23}$ l'héritage dionysien dans la théologie de l'Église terrestre post-tridentine : l'analogie entre Église terrestre et Église céleste, qui repose chez le pseudo-Denys sur la relation de similitude unissant hiérarchie céleste (archanges, anges) et hiérarchie humaine, légale puis ecclésiastique (évêques, prêtres, ministres ou diacres) ${ }^{24}$; le rôle des médiateurs, qui permettent d'amener les êtres inférieurs vers les êtres supérieurs, et en définitive vers Dieu; l'idée que l'illumination est fondamentalement descendante, les médiateurs ne pouvant illuminer les inférieurs que s'ils sont eux-mêmes illuminés par des médiateurs plus élevés; enfin (dans une certaine mesure du moins), l'idée - issue de la lecture du pseudo-Denys par Thomas d'Aquin - qu'au sommet de cette hiérarchie se trouve le pape, hierarchiae praecipuus ou summus, évêque par excellence ${ }^{25}$, doté de la plénitude du pouvoir ${ }^{26}$.

\section{DES INFLÉCHISSEMENTS SIGNIFICATIFS}

Bossuet hérite cependant de Bérulle un premier infléchissement de l'ecclésiologie dionysienne : alors que chez le pseudo-Denys, le hiérarque couronnait une hiérarchie de connaissance ou d'illumination $^{27}$, chez Bossuet, comme chez Bérulle ${ }^{28}$, il surplombe une hiérar-

ordonné d'un cosmos, par leur « rôle » dans la hiérarchie dynamique selon laquelle tout est référé à un 'centre' » (La Spiritualité de Bossuet, p. 95).

23. Yves Congar, L'Église de saint Augustin à l'époque moderne, Paris, Cerf, 1970, p. 225.

24. Cette idée n'est nullement propre au corpus dionysien, mais celui-ci lui fournit un puissant appui.

25. Voir René Roques, L'Univers dionysien. Structure hiérarchique du monde selon le Pseudo-Denys, Paris, Cerf, 1983, p. 182 : «Pierre est un premier (coruphaios) parmi des égaux (homotagous); mais Denys ne définit pas la nature d'une pareille primauté, ni ses conditions d'exercice, ni les lois qui, éventuellement, pourraient en régler la transmission. »

26. Ibid., p. 228.

27. Même lorsque, après avoir été introduit en latin par la tradition de Jean Scot Érigène, le terme hierarchia sera utilisé par les héritiers du pseudo-Denys (tels que Bonaventure), il n'équivaudra pas exactement à ce que nous appelons aujourd'hui «la hiérarchie » (institutionnelle), et continuera d'abord à désigner la hiérarchie spirituelle des initiés. Voir Yves Congar, L'Église de saint Augustin à l'époque moderne, Paris, Cerf, 1970, chap. XII, « Ecclésiologie de la Contre-Réforme », p. 369, n. 2.

28. Voir Michel Dupuy, Bérulle et le sacerdoce, p. 70 : "Pour Bérulle, le prêtre est médiateur de la foi en raison de son "autorité", alors que pour Denys il 
chie de pouvoir ${ }^{29}$ : plus encore que le plus savant, le plus éclairé, il est le plus puissant, celui qui est doté de la puissance maximale. C'est ainsi que dans le sermon d'ouverture de l'Assemblée du clergé de 1681 (dit Sermon sur l'unité de l'Église), fortement teinté, on l'a $\mathrm{vu}$, de dionysisme, Dieu est présenté comme la source de la puissance unique des ecclésiastiques, qui s'en voient déléguer une part plus ou moins importante selon leur rang, depuis la «plénitude de la puissance apostolique » (plenitudo potestatis) conférée au pape jusqu'à la moindre puissance conférée aux prêtres et aux ministres (ou diacres) :

«Tous reçoivent la même puissance, et tous de la même source, mais non pas tous en même degré, ni avec la même étendue, car JésusChrist se communique en telle mesure qu'il lui plaît, et toujours de la manière la plus convenable à établir l'unité de son Église ${ }^{30}$. »

On note encore chez le gallican Bossuet deux infléchissements supplémentaires de l'ecclésiologie dionysienne : d'une part, le thème de la plenitudo potestatis du pape n'apparait - on le conçoit aisément qu'épisodiquement; d'autre part, Bossuet n'emprunte que partiellement au pseudo-Denys sa conception des médiations successives et n'insiste que ponctuellement sur les divers degrés de l'autorité ecclésiastique (d'abord purification, puis illumination, puis perfection ${ }^{31}$ ); plus largement du reste, dans le domaine religieux comme dans le domaine politique, Bossuet ne se montre guère intéressé par les corps intermédiaires, et en cela s'avère profondément absolutiste.

Or, si, contrairement au pseudo-Denys, Bossuet n'est guère enclin à un partage strict des « domaines de compétences » (purifi-

"illumine" grâce à sa science qui perçoit la signification des signes sacrés [...]; il faut traiter de la transmission de l'autorité par succession dans l'histoire, alors que le Pseudo-Denys s'intéresse surtout à la transmission de la "lumière" par diffusion, en faisant abstraction de la dimension temporelle. »

29. Non sans paradoxe, il en va de même pour Henry Holden, inspirateur majeur de Richard Simon, auquel, comme on sait, Bossuet s'opposa violemment. Sur les notions de hiérarchie et d'institution chez Holden, voir Jacques Le Brun, «L'institution dans la théologie de Henry Holden (1596-1662)», Recherches de science religieuse, janvier-mars 1983, t. $71, \mathrm{n}^{\circ} 1, \mathrm{p} .201$. Il est pourtant difficile de savoir si Bossuet a lu Holden : une influence semble perceptible, mais aucun volume de Holden n'a été relevé dans les catalogues - incomplets - de la bibliothèque de Bossuet.

30. Bossuet, Sermon sur l'unité de l'Église (1681), in O.O., t. VI, p. 115.

31. Autrement dit, Bossuet ne reprend pas formellement la triade de l'activité théarchique définie par le pseudo-Denys (purification, illumination, union), même si des réminiscences en sont ponctuellement perceptibles dans son œuvre. 
cation, illumination, perfection), c'est qu'il se montre plus sensible à la définition « englobante » de la fonction des évêques, idée également présente chez l'Aréopagite, parallèlement à la tripartition des fonctions, et selon laquelle les ordres supérieurs de la hiérarchie ecclésiastique détiennent en les transcendant les fonctions des ordres inférieurs. Dans cette perspective, les champs d'exercice ne sont pas nettement séparés et complémentaires, mais bien plutôt concentriques; autrement dit, loin que les trois ordres entretiennent une relation de stricte complémentarité, ils se comprennent comme participations inégales à un même sacerdoce. Non seulement les ordres inférieurs ne peuvent remplir les fonctions dévolues aux ordres supérieurs, mais loin d'accomplir un sacerdoce qui leur serait spécifique et réservé, ils ne peuvent remplir leur mission qu'en union avec l'évêque et sous sa dépendance, puisque ce dernier est, par excellence, le dépositaire du divin parmi les hommes ${ }^{32}$. Quant au prêtre, il n'est pour Bossuet que « coopérateur de l'épiscopat » ${ }^{33}$.

En dépit de ces infléchissements sensibles, entre M. de Meaux et le pseudo-Denys, les analogies demeurent cependant bien plus frappantes que les différences. L'évêque, « lumière ardente et luisante ", déclare dans un style très dionysien l'auteur du Panégyrique de saint François de Sales, est « établi dans le premier ordre de la dignité ecclésiastique ${ }^{34}$; il constitue ainsi le relais le plus important dans la transmission de l'autorité et de la doctrine divines, non seulement dans l'ordre synchronique (depuis les plus hautes instances ecclésiastiques jusqu'aux fidèles), mais dans l'ordre diachronique (depuis les apôtres jusqu'à l'époque moderne). Comme l'indique en effet clairement le Catéchisme du diocèse de Meaux que Bossuet rédige à l'intention de ses jeunes diocésains, ce sont les évêques qui se sont transmis successivement la doctrine chrétienne telle qu'elle avait été reçue par les apôtres :

«Pourquoi dit-on que l'Église est apostolique? / Parce que les évêques ou principaux pasteurs ont succédé sans interruption aux apôtres. / Qu'appelez-vous sans interruption? / En s'ordonnant et consacrant successivement les uns les autres, depuis le temps des apôtres

32. Voir R. Roques, L'Univers dionysien. Structure hiérarchique du monde selon le Pseudo-Denys, op. cit., p. 184.

33. Bossuet, Oraison funèbre du Père Bourgoing (1662), in O.O., t. IV, p. 416.

34. Bossuet, Panégyrique du bienheureux saint François de Sales (1660), in O.O., t. III, p. 585 . 
jusqu'à nous, sans interruption. / Pourquoi cette succession?/ Pour transmettre successivement, et comme de main en main, la doctrine apostolique, depuis le temps des apôtres, jusqu'à la fin du monde ${ }^{35}$. »

\section{UN DIONYSISME ÉPISCOPAL}

Ce que Bossuet retient donc essentiellement du pseudo-Denys, en dehors de la conception générale d'un monde étagé en hiérarchies successives, c'est sa méditation sur la hiérarchie ecclésiastique, « cité du divin », et en particulier sur l'importance de l'épiscopat: non seulement l'ordre des évêques est le premier parmi les ordres qui voient Dieu, mais il constitue en quelque sorte l'alpha et l'oméga de la hiérarchie ecclésiastique, «car en lui s'accomplit et s'achève toute ordonnance de la hiérarchie humaine $»^{36}$. Pour Bossuet comme pour le platonicien Denys, l'évêque est tout naturellement, ontologiquement et chronologiquement, le premier agent des sacrements, puisqu'il est le seul à pouvoir ordonner prêtres et ministres (ou diacres) : de même que chez Platon, les philosophes-rois constituent en quelque sorte la quintessence de la classe des gardiens dont les autres membres ne figurent que des versions imparfaites et ancillaires, l'évêque se situe dans un rapport d'accomplissement et de souveraineté par rapport aux autres initiateurs ${ }^{37}$. Cette prééminence semble plus marquée encore chez Bossuet que chez le pseudo-Denys : pour l'évêque de Meaux en effet, l'Église se présente à bien des égards comme une « théocratie » centralisée dont les évêques, tout-puissants dans leurs diocèses respectifs, sont les ministres et les garants d'unité; comme il l'écrit dans l'Oraison funèbre du Père Bourgoing (et cela est d'autant plus significatif que le défunt n'avait jamais été évêque) :

« Le mystère de l'autorité ecclésiastique est dans la personne, dans le caractère, dans l'autorité des évêques. En effet, Chrétiens, ne voyezvous pas qu'il y a plusieurs prêtres, plusieurs ministres, plusieurs prédicateurs, plusieurs docteurs; mais il n'y a qu'un seul évêque dans un diocèse et dans une église ${ }^{38}$. »

35. Bossuet, Catéchisme du diocèse de Meaux (second catéchisme), in O.C. (pour Euvres complètes, éditées par F. Lachat, 31 vol., Paris, Louis Vivès, 18621866), vol. V, p. 69.

36. Pseudo-Denys, Hiérarchie ecclésiastique, 505 A.

37. Ibid., p. 86.

38. Bossuet, Oraison funèbre du Père Bourgoing, supérieur de l'Oratoire (1662), O.O., IV, p. 414. 
L' "ordre ecclésiastique» dionysien, où l'évêque assumait une fonction éminente, mais intégrée dans toute une hiérarchie, fait place chez Bossuet à un « ordre épiscopal », où n'apparaissent plus guère que le pape et les évêques, et où les prêtres, "établis de Dieu », on l'a vu, «pour être coopérateurs de l'épiscopat $»^{39}$, se voient cependant assigner une fonction clairement subalterne.

Ainsi conçue, l'autorité épiscopale englobe tous les types de médiation ecclésiastique : administration des sacrements bien sûr (à l'évêque étant notamment réservée l'administration du sacrement de la confirmation et de l'ordre ${ }^{40}$ ), mais aussi prédication ${ }^{41}$, ainsi que les deux pouvoirs propres à l'évêque (terme dont l'étymologie, comme on sait, signifie : le surveillant), l'ordre et la juridiction ${ }^{42}$.

C'est enfin à l'évêque, ou plutôt au «saint concours des évêques » ${ }^{43}$, de protéger le «dépôt de la foi ». Les occurrences de ce thème sont nombreuses chez Bossuet ${ }^{44}$, en particulier dans les textes de controverse, que ces controverses soient internes ou externes au catholicisme. Dans l'Instruction sur la version du Nouveau Testament imprimée à Trévoux par exemple, Bossuet écrivant

39. Ibid., p. 416.

40. Sur l'importance que Bossuet accorde, dans sa pratique, à l'ordination, voir M. Bardon, Bossuet à Meaux. La vie de Bossuet dans son diocèse, op. cit., p. 53.

41. Alors même que bien des évêques du temps ne prenaient pas la peine de prêcher, Paul Lesourd indique que dès son premier sermon comme évêque de Meaux, Bossuet promit « qu'il prêcherait lui-même toutes les fois qu'il officierait pontificalement » («Bossuet, évêque de Meaux », Revue des jeunes, 1927, p. 325) ce qu'il fit en effet.

42. Dans l'Histoire des Variations, Bossuet voit dans les devoirs « de visiter et de corriger » « la véritable fonction épiscopale » (Histoire des variations in O.C., vol. XIV, p. 180). De même, dans les Instructions sur la version du Nouveau Testament imprimée à Trévoux, invoquant les décrets tridentins, il rappelle que les évêques « sont chargés par le concile de déclarer, c'est-à-dire de noter les contrevenants à sa règle, et même de les punir » (Instruction sur la version du Nouveau Testament imprimée à Trévoux, addition sur la remontrance de M. Simon à Mgr le Cardinal de Noailles, in O.C., vol. III, p. 452).

43. Voir Bossuet, Relation sur le quiétisme, VI, 7, in O.C., vol. XX, p. 144 : « J'enseignais la théologie de toute l'Eglise : l'approbation de M. de Paris et celle de M. de Chartres y ajoutaient l'autorité que donne naturellement dans les matières de la foi, le saint concours des évêques »".

44. Voir par exemple le Sermon sur les devoirs des rois (1662), in O.O., t. IV, p. 367. On observera du reste que pour Bossuet, toute autorité - et a fortiori toute autorité ecclésiastique - relève du dépôt, et non de la propriété. Voir l'Oraison funèbre de Michel Le Tellier, in O.O., t. IV, p. 330 : «Enfin dans une heureuse vieillesse, prêt à rendre avec sa grande âme le sacré dépôt de l'autorité si bien confié à ses soins... ». 
en tant que « Monseigneur l'illustrissime et révérendissime évêque de Meaux » déclare : « il nous suffira de proposer la vérité, dont le précieux dépôt est confié aux évêques ${ }^{45}$ ». De même, le texte intitulé Sur la remontrance de M. Simon à Mgr le Cardinal de Noailles comporte la question rhétorique suivante : « Mais à qui conviendrat-il mieux de relever de telles fautes, qu'aux évêques qui sont chargés du dépôt des Écritures $?^{46}{ }^{\prime \prime}$

Il est du reste significatif que, faisant appel en 1702 à l'autorité de l'évêque de Meaux contre Port-Royal, Étienne de Champflour lui parle son langage, ouvrant sa lettre par la même expression topique, qui semble décalquée du discours bossuétiste et ne peut que flatter le sentiment exacerbé que Bossuet possède de son autorité épiscopale :

«La bonté que vous m'avez témoignée autrefois, pendant que je demeurais dans le séminaire de Saint-Sulpice, me fait espérer que vous ne trouverez pas mauvais que $\mathrm{j}$ 'aie recours à votre protection dans l'occasion présente, où il s'agit de la conservation du sacré dépôt de la foi, dont les évêques sont chargés spécialement, et que je sais vous être si à cœur ${ }^{47}$. »

Comme le philosophe-roi de Platon, l'episcopus est bien pour Bossuet un gardien.

Et ce gardien doit être aussi irréprochable que son dépôt est précieux : cette hypervalorisation de l'épiscopat va donc de pair avec une conception très exigeante de ses devoirs. Chez Bossuet, les devoirs de l'évêque - proclamés par le pseudo-Denys puis rappelés dans le droit canon (notamment dans le décret de Gratien ${ }^{48}$ ), et dans des «miroirs de l'évêque » ainsi que dans des ouvrages de spiritualité épiscopale de plus en plus nombreux au XVII ${ }^{\mathrm{e}}$ siècle $^{49}-$ se trouvent notamment évoqués par l'intermédiaire du portrait

45. Bossuet, Instruction sur la version du Nouveau Testament imprimée à Trévoux, in O.C., vol. III, p. 381.

46. Bossuet, Sur la remontrance de M. Simon à Mgr le Cardinal de Noailles, $6^{\text {e }}$ remarque, $\S 4$, in $O . C$., vol. III, p. 473.

47. Lettre d'Étienne de Champflour à Bossuet, 20 novembre 1702, in Bossuet, Correspondance, t. XIV, p. 22-23.

48. Voir M. Peronnet, Les Évêques de l'ancienne France, op. cit., p. 399.

49. Par exemple, le Manuel de l'activité épiscopale (Episcopalis sollicitudinis enchiridion) de Louis Abelly, évêque de Rodez, publié en 1668, réflexion sur l'œuvre de Charles Borromée. De même, aux auctoritates régulièrement invoquées, telles que saint Paul, saint Chrysostome, Grégoire le Grand (Regula Pastoralis), Barthélémy des Martyrs, archevêque de Braga (et son Stimulus pastorum). 
d'évêques réformateurs tels que Charles Borromée et François de Sales ${ }^{50}$.

Or, fait remarquable, le Panégyrique que consacre Bossuet en 1660 à François de Sales, " saint évêque " ${ }^{51}$ demeure très imprégné de dionysisme. Il évoque successivement dans ses trois points la science de François docteur, l'autorité de François évêque et la douceur de François directeur de conscience, et ces trois vertus, se trouvent unifiées autour de la métaphore topique - mais aussi, plus spécialement, dionysienne - de la lumière :

« La science l'a rendu un flambeau capable d'illuminer les fidèles; la dignité épiscopale a mis le flambeau sur le chandelier pour éclairer toute l'Eglise; et le soin de la direction a appliqué cette lumière bénigne à la conduite des particuliers ${ }^{52}$. »

Cependant, si le Panégyrique du Bienheureux saint François de Sales apparaît très empreint de dionysisme, il met également l'accent sur un autre thème, qui était déjà prégnant dans le Panégyrique que Bossuet consacrait, quelques années auparavant, à Charles Borromée, archevêque de Milan entre 1563 et 1584 et lui aussi modèle épiscopal typique de la Contre-Réforme ${ }^{53}$; il s'agit du positionnement paradoxal de l'évêque, entre grandeur et abaissement, annoncé dès l'exorde ${ }^{54}$ :

«Ces trois qualités [intercesseurs, princes et pères du peuple] les mettent certainement au-dessus de tous en qualité de pontifes; mais,

50. Plus largement, de nombreux modèles étaient proposés aux évêques à travers des Vies de pasteurs anciens tels que Denys le Chartreux, Laurent Justinien, Antonin.

51. Bossuet, Panégyrique du Bienheureux saint François de Sales (1660), in O.O., t. III, p. 579 et p. 591 .

52. Ibid., p.578. Si l'on se rappelle que l'illumination désignait chez le pseudo-Denys le but de l'action des prêtres, cette citation confirme d'une part que Bossuet voit dans l'évêque un prêtre plus accompli, sans solution de continuité avec le reste de l'ordre sacerdotal; d'autre part qu'il ne se montre guère sensible à la tripartition bien différenciée des fonctions élaborée par le pseudo-Denys (voir supra).

53. Son influence, comme celle de la plupart des modèles épiscopaux, s'exerce par l'intermédiaire de nombreux supports : les Acta Ecclesiae mediolanensis, les Instructions aux confesseurs (souvent rééditées), les biographies, l'iconographie. Voir J. Le Brun, art. «France. XVII siècle; le Grand siècle de la spiritualité française et ses lendemains », Dictionnaire de spiritualité ascétique et mystique, t. 5, col. 927.

54. Nous ne connaissons malheureusement ce discours que par des notes prises à l'audition, et publiées dans l'édition des O.O., t. II, p. 575-594. 
pour accomplir ce ministère, si elles les élèvent au-dessus de tous en qualité de pontifes, elles les abaissent au-dessous de tous en qualité de victimes ${ }^{55}$. »

Face au danger d'autoritarisme induit par la hiérarchisation de plus en plus grande de l'Église, le panégyriste proclame ainsi par l'intermédiaire de l'exemplum borroméen que l'évêque, à l'image de Paul, trouve dans sa faiblesse même sa force. L'autorité pastorale est une autorité paradoxale fondée sur l'humilité, thème paulinien qui prend chez Bossuet une importance qu'il ne possédait pas chez le pseudo-Denys : «Ah! la tyrannie des prêtres, c'est leur faiblesse et leur infirmité56 ${ }^{\prime \prime}$, formule qui reviendra dans la Politique tirée des propres paroles de l'Écriture sainte à propos d'Ambroise, évêque de Milan ${ }^{57}$. Entre ciel et terre, entre force et faiblesse, l'évêque joue par excellence, selon Bossuet, le rôle toujours paradoxal d'un médiateur, fort de l'autorité qu'il porte :

« Il faut encore écouter Dieu parler par le ministère des supérieurs, qui vous représentent Jésus-Christ, et spécialement dans les visites pastorales, où le Saint-Esprit préside infailliblement ${ }^{58} »$,

et faible de sa propre nature - radicalement étrangère à cette autorité, et indigne d'elle :

« De nous-mêmes, nous ne saurions conférer aux autres la moindre grâce; c'est Dieu, comme dit l'Apôtre, qui nous en rend capables. Et vous voyez par là combien vous êtes intéressées à demander pour nous à l'auteur de tout don, qu'il prépare nos cœurs et les vôtres, afin que nous puissions produire des fruits abondants parmi vous ${ }^{59}$.»

55. Bossuet, Panégyrique de saint Charles Borromée (1656), in O.O., t. II, p. 578.

56. Ibid.

57. De même, la Politique présentant Ambroise comme le modèle de l'évêque obéissant au pouvoir temporel sans déroger à son autorité spirituelle, lui mettra en la bouche cette parole : «Puisqu'on appelle cela une tyrannie, j'ai des armes, disait-il; j'ai le pouvoir d'offrir mon corps en sacrifice. Nous avons notre tyrannie et notre puissance. La puissance d'un évêque est sa faiblesse. Je suis fort quand je suis faible, disait saint Paul ».

58. Bossuet, Troisième exhortation aux Ursulines de Meaux (1685), in O.O., t. VI, p. 242. Un peu plus loin, les ministres sont identifiés aux « oints du Seigneur » que l'Écriture ordonne de respecter, et mention est faite de «leur grand pouvoir et leur sublime dignité » (ibid., p. 243). Voir aussi ibid., p. $244:$ « $\mathrm{Ne}$ regardez que l'autorité que Dieu a donnée sur vous à ses ministres ».

59. Ibid., p. 272. 
Une étude de l'utilisation proprement rhétorique ${ }^{60}$ - et en particulier éthique, au sens où l'ethos désigne l'image que le locuteur construit de lui-même à travers son discours - du thème de l'épiscopat, montrerait que cette ambivalence est inscrite au cœur même de l'idée que se fait l'évêque de Meaux de son autorité ${ }^{61}$. Elle constitue peut-être l'infléchissement majeur que Bossuet fait subir à l'héritage dionysien - qui conserve cependant une importance capitale dans l'ecclésiologie d'un auteur représentatif en cela à la fois de la Réforme catholique dans son ensemble et des préoccupations gallicanes plus spécifiques à son milieu : la médiation n'est plus seulement pensée en termes d'ordre et de beauté lumineuse, mais en termes de force et de faiblesse, d'autorité et d'humilité. La hiérarchie solaire du pseudo-Denys est devenue un clair-obscur.

anne.regent-susini@univ-paris3.fr

60. Sur ce point, je me permets de renvoyer à mon ouvrage à paraître : Bossuet et la rhétorique de l'autorité : "voix devant la Parole », Paris, Honoré Champion (« Lumière classique »).

61. Sur la conception de l'autorité développée par Bossuet, je me permets de renvoyer à mon ouvrage à paraître : Le Centre et la Ligne. La pensée de l'autorité dans l'œuvre de Bossuet, Berlin, Lit Verlag (« Ars rhetorica »). 